\title{
H.C.Diener
}

\section{Schwerpunktthema „Kopfschmerz“}

Das Thema dieses Heftes beschäftigt sich mit neuen wissenschaftlichen und klinischen Aspekten des Kopfschmerzes. Es wurde mit Absicht darauf verzichtet, die neuen gängigen Therapieverfahren bei der Migräne darzustellen, da dies in den letzten Jahren ausführlich an anderer Stelle erfolgte. Die ersten Artikel beschäftigen sich mit den neuen Tiermodellen und den neuen experimentellen An-

H.C. Diener, Neurologische Universitätsklinik Hufelandstraße 55, D-45122 Essen sätzen zur Quantifizierung von Kopfschmerzen und zu neuen Einsichten in die Pathophysiologie von Kopfschmerzen beim Menschen. In einer kritischen Übersicht wird versucht herauszustellen, bei welchen Patienten und in welchen Situationen Bildgebung und andere technische Zusatzuntersuchungen erforderlich oder entbehrlich sind. Drei Artikel widmen sich schwierigen bzw. kontroversen Therapien, nämlich der Behandlung der menstruellen Migräne, dem Einsatz der Homöopathie und der Akupunktur bei Kopfschmerzen. Ich hoffe, daß die Themenauswahl sowohl dem wissenschaftlich Interessierten wie auch dem klinisch Tätigen umfassende neue Informationen bietet.

\section{V.Pfaffenrath}

\section{Epidemiologie und Kopfschmerz - zum kritischen Umgang mit Daten}

54 Millionen Deutsche mit Kopfschmerzen? Vier Milliarden DM direkte und indirekte Kosten für die Betreuung von Migränepatienten? Volkskrankheit Kopfschmerz? Der resignierte Patient? Auf den ersten Blick liest sich das wie Schlagzeilen aus der Regenbogenpresse, obwohl es sich um Zitate aus der medizinischen Literatur oder aus Pressekonferenzen handelt. Wird mit solchen Daten Politik gemacht? Früher dienten epidemiologische Studien ausschließlich der Identifikation einer Krankheitsätiologie oder es konnten bestimmte ärztliche Behandlungsmuster aufgezeigt werden in Abhängigkeit z. B. davon, ob überhaupt ein Arzt aufgesucht wurde, oder ob erst eine andere Erkrankung zur Diagnose z. B. einer Migräne führte. In den letzten Jahren verfolgten solche Untersuchungen das fast ausschließliche Ziel, Daten zur Prävalenz zu erhalten. Für die Migräne (nach der Definition der IHS) geht man heute von einer Lebenszeitprävalenz von ca. $10 \%$ aus. Eine Lebenszeitprävalenz der Migräne schließt also auch solche Patienten ein, die nur einmal in ihrem Leben eine Migräneattacke gehabt haben. Darüberhinaus wurden trotz der bekannten Nachteile Fragebögen statt eines semistrukturierten Interviews verwendet. Ein rating der Symptome, z. B. ob Übelkeit leicht oder schwer ist, ist nicht obligat, obwohl gerade dies in den letzten beiden Jahren zur Unterscheidung der Migräne vom Spannungskopfschmerz propagiert wird. Nicht vergessen werden sollte, daß viele Migräniker keinen Arzt konsultieren und deswegen Studien, die sich auf medizinische Diagnosen oder Selbstdiagnosen stützen, üblicherweise die Migräneprävalenz unterschätzen. Darüberhinaus ist die Migräneprävalenz abhängig von Alter, Geschlecht, Rasse und Sozialfaktoren wie z. B. Einkommen.
Fast immer handelt es sich um eine Querschnittbefragung ohne Daten zur Vorgeschichte. Noch weniger wird darauf eingegangen, $\mathrm{da}$ die Symptomatologie einer Migräne sich mit zunehmendem Lebensalter und unter Therapie ändern kann. Darüberhinaus sind die untersuchten Populationen in den wenigsten Fällen miteinander vergleichbar. Erinnert sei in diesem Zusammenhang an eine Studie [1] im Zusammenhang mit Sumatriptan, mit dem Ziel, die direkten Kosten der Migräne zu berechnen, wobei ausschließlich Studienpatienten, in einem Fall selektiv kanadische Krankenschwestern mit einem Durchschnittsalter von 30 Jahren eingeschlossen wurden. Die fehlende Berücksichtigung dieser Variablen bei epidemiologischen Studien dürfte mitverantwortlich sein für die unterschiedlichen in der Literatur angegebenen Prävalenzen. So schwankte in einer Metaanalyse von Lipton über 24 Stunden die Prävalenz über ein Jahr bzw. die Lebenszeit und bei verschiedenen Altersgruppen zwischen 3,2 und 48,3\% [2].

Es mag deshalb erstaunen, da $B$ manche der Prävalenzdaten zur Migräne vor Einführung der IHS-Kriterien in erstaunlich guter Übereinstimmung sind mit den Daten, die erhalten wurden, als die IHS-Definition weltweit auf ihre Reliabilität und Validität untersucht wurden. Acht Millionen Migräniker mit einem Frequenzspektrum der Attacken von $1 \times /$ Jahr bis $6 \times /$ Monat und drei Millionen Patienten mit einem fast täglichen Spannungskopfschmerz rechtfertigen es allerdings nicht, aus gesundheitspolitischen oder dramaturgischen Gründen statistische Zahlenspiele zu betreiben und nahezu 2/3 der Bevölkerung dieses Landes zu Kopfschmerzkranken zu stempeln. Dankenswerterweise tragen die Beiträge in diesem Sonderheft zur Versachlichung der Thematik bei.

\section{Literatur}

1. Osterhaus JT, Guttermann DL, Plachetka JR (1992) Healthcare resource and lost labour costs of migraine headache in the US. Pharmaco Economics: 2: 67

\section{Lipton R, Silberstein SD, Stewart WF}

(1994) An update on the epidemiology of migraine. Headache 34: 319

V.Pfaffenrath, Leopoldstraße 59/II, D-80802 München 